Pacific Journal of Mathematics

ON THE COMPLETION OF LOCALLY SOLID VECTOR 


\title{
ON THE COMPLETION OF LOCALLY SOLID VECTOR LATTICES
}

\author{
D. H. FREMLIN
}

\begin{abstract}
Let $E$ be a Riesz space (= vector lattice), with a locally solid Hausdorff linear space topology. Then its completion also has a Riesz space structure. In this paper it is shown how a pair of important properties which may be possessed by $E$ are inherited by its completion.
\end{abstract}

In general this article will rest on the foundations of [4] and [5]. A linear space topology on a Riesz space $E$ is locally solid if 0 has a neighbourhood basis consisting of solid sets. In this case, the lattice operations are uniformly continuous; consequently (assuming that the topology is Hausdorff) they can be extended to the linear topological space completion $\hat{E}$ of $E$, and $\hat{E}$ will also be a locally solid topological Riesz space $([5$, p. $235 ; 4$, p. 108]). $E$ is now a Riesz subspace of $\hat{E}$, i.e. a linear subspace which is also a sublattice.

My object is to show how two important and common properties are preserved by the process of completion. Unfortunately, although these properties have been studied by various authors (see e.g. [3]), no satisfactory terminology has been devised. I hope that my use of the words "Fatou" $(\S 1)$ and "Lebesgue" $(\S 5)$, suggested by the famous convergence theorems, will prove acceptable.

1. Fatou topologies. Let $E$ be a Riesz space and $\mathfrak{I}$ a topology on $E$. I will call $\mathfrak{I}$ Fatou if (i) it is a linear space topology (ii) 0 has a base consisting of sets $U$ which are solid and such that if $\varnothing \subset A \subseteq U$ and $A \uparrow x$ in $E$ (i.e. if $A$ is nonempty, directed upwards, and has $x$ for its least upper bound), then $x \in U$.

This property is exceedingly common. Consider, for example, $C(X)$ for any compact space $X$; the basic neighbourhoods of 0 are of the form $\left\{x:\|x\|_{\infty} \leqq \varepsilon\right\}$, and these all have the property described above. Similarly, in all the $L^{p}$ spaces, for $0 \leqq p \leqq \infty$, the usual topologies are Fatou.

The most striking thing about Fatou topologies is Nakano's theorem (see [2]). For its full strength this requires a further concept. Let us call a linear space topology on a Riesz space $E$ a Levi topology if every topologically bounded set $A \subseteq E$ which is directed upwards has an upper bound in $E$. (For example, all the spaces adduced above have Levi topologies. Also, the weak topology associated with a locally convex Hausdorff Levi topology will always be Levi). Then: A Levi Fatou Hausdorff topology on a Dedekind complete Riesz space is com- 
plete. For a proof of this theorem, see [4], Proposition IV. 1.5. ([4] uses the phrases "locally order complete" and "boundedly complete" for Fatou and Levi topologies respectively in Dedekind complete spaces).

2. Extensions of Riesz spaces; the spaces $C_{\infty}(X)$. Let $E$ be a Riesz space. I shall call a Riesz subspace $F$ of $E$ orderdense if, for every $x \geqq 0$ in $E$,

$$
x=\sup \{y: y \in F, 0 \leqq y \leqq x\} .
$$

An important consequence of this is that if $A$ is a nonempty subset of $F$ and $x=\sup A$ in $F$, that is, if $x$ is the least member of $F$ which is an upper bound of $A$, then $x=\sup A$ in $E$. It follows that if $F$ is orderdense in $E$, and $G$ is orderdense in $F$, then $G$ is orderdense in $E$.

Let $X$ be a compact extremally disconnected Hausdorff topological space. Let $C_{\infty}(X)$ be the set of all those continuous functions $x$ from $X$ to the extended real line $[-\infty, \infty]$ such that $\{t:-\infty<x(t)<\infty\}$ is dense in $X$. Because every continuous real-valued function defined on a dense open subset of $X$ has a unique extension to a member of $C_{\infty}(X)$ ([6, Lemma V. 2.1]), $C_{\infty}(X)$ has a natural Riesz space structure under which it is Dedekind complete ([6, Theorem V. 2.2]). The point is that every Archimedean Riesz space can be embedded as an orderdense Riesz subspace of some $C_{\infty}(X)$ ([6, Theorems IV. 11.1 and V. 4.2]).

[6] gives several properties of the space $C_{\infty}(X)$, but not the one we shall need; so I set it out here.

Proposition 1. Let $X$ and $C_{\infty}(X)$ be as above. Let $A \leqq C_{\infty}(X)^{+}$ be a nonempty set such that for every $x>0$ in $C_{\infty}(X)$ there is an $n \in N$ such that

$$
n x \neq \sup _{y \in A} y \wedge n x .
$$

Then $A$ is bounded above in $C_{\infty}(X)$.

Proof. Define $w: X \rightarrow[0, \infty]$ by

$$
w(t)=\sup _{y \in A} y(t) \forall t \in X .
$$

Then $w$ is lower semi-continuous. Define $v: X \rightarrow[0, \infty]$ by

$$
v(t)=\inf \left\{\sup _{u \in U} w(u): U \text { a nhd of } t\right\}
$$


for every $t \in X$. Then $v$ is continuous ([6, Theorem V.1.1]). My aim is to prove that $v \in C_{\infty}(X)$, i.e. that $v$ is finite on a dense set.

Suppose that $G \subseteq X$ is open and not empty. As $X$ is compact and Hausdorff, there is a continuous function $x$ on $X$ such that $x>0$ but $x(t)=0 \forall t \in X \backslash G$. Now $x \in C_{\infty}(X)$, so there is an $n \in N$ such that

$$
n x \neq \sup _{y \in A} y \wedge n x
$$

that is, there is a $z>0$ in $C_{\infty}(X)$ such that

$$
y \wedge n x \leqq n x-z \forall y \in A .
$$

Of course $z \leqq n x$, so $z$ is finite everywhere and $z(t)=0 \forall t \in X \backslash G$. Let $H=\{t: z(t)>0\}$; then $H$ is not empty and $H \subseteq G$.

But if $t \in H, y(t) \leqq n x(t)-z(t) \forall y \in A$, so $w(t) \leqq n x(t)-z(t)$; and as $n x-z$ is continuous, $v(t) \leqq n x(t)-z(t)<\infty \forall t \in H$.

Consequently, $\{t: v(t)<\infty\}$ meets $G$. As $G$ is arbitrary, $v \in C_{\infty}(X)$ and is the required upper bound for $A$.

3. THEOREM 1. Let $E$ be an Archimedean Riesz space with a Hausdorff Fatou topology. Let $\hat{E}$ be its linear topological space completion with its natural Riesz space structure. Then (i) $E$ is an orderdense Riesz subspace of $\hat{E}$ (ii) the topology on $\hat{E}$ is Fatou.

Proof. My method is to find a complete Riesz space extending $E$ which has the required properties.

(a) Let $X$ be a compact extremally disconnected Hausdorff topological space such that $E$ can be embedded as an orderdense Riesz subspace of $C_{\infty}(X)$ ( $\S 2$ above). Let $\mathscr{B}$ be the set of all neighbourhoods $U$ of 0 in $E$ satisfying the Fatou property in $\S 1$, i.e. such that $U$ is solid and if $\varnothing \subset A \subseteq U$ and $A \uparrow x$ in $E$ then $x \in U$. Then $\mathscr{B}$ is a base of neighbourhoods of 0 . For each $U \in \mathscr{B}$, set

$$
\widetilde{U}=\left\{w: w \in C_{\infty}(X), \forall x \in E,|x| \leqq|w| \Rightarrow x \in U\right\} .
$$

Then $\tilde{U}$ is a solid subset of $C_{\infty}(X)$. Note that $\tilde{U} \cap E=U$.

(b) Suppose that $U$ and $V$ belong to $\mathscr{B}$ and that $U+U \subseteq V$. Then $\widetilde{U}+\widetilde{U} \subseteq \tilde{V}$. For suppose that $w_{1}, w_{2} \in \widetilde{U}$ and that $x \in E$ is such that $|x| \leqq\left|w_{1}+w_{2}\right|$. Set $v_{1}=\left|w_{1}\right| \wedge|x|$ and $v_{2}=|x|-v_{1} \leqq\left|w_{2}\right|$. Then $A_{i}=\left\{y: y \in E, 0 \leqq y \leqq v_{i}\right\} \uparrow v_{i}$ for $i=1,2$, so $A_{1}+A_{2} \uparrow v_{1}+v_{2}=$ $|x|$ in $E$. But $A_{1}+A_{2} \subseteq U+U \subseteq V$, so $|x| \in V$ and $x \in V$. As $x$ is arbitrary, $w_{1}+w_{2} \in \tilde{V}$; as $w_{1}$ and $w_{2}$ are arbitrary, $\widetilde{U}+\widetilde{U} \subseteq \tilde{V}$.

(c) It follows that if we set 


$$
H=\bigcap_{U \in \mathscr{S}} \bigcup_{\alpha \in \mathscr{R}} \alpha \widetilde{U}
$$

then $H$ is a solid linear subspace of $C_{\infty}(X)$, including $E$, and $\{\widetilde{U} \cap H$ : $U \in \mathscr{B}\}$ is a neighbourhood basis at 0 for a linear space topology $\mathfrak{T}$ on $H$. As every $\widetilde{U} \cap H$ is solid, $\mathfrak{I}$ is locally solid; as $\widetilde{U} \cap E=U$ for every $U \in \mathscr{B}, \mathfrak{I}$ induces the original topology on $E$. Also, $\mathfrak{I}$ is Hausdorff, for if $w \in H$ and $w \neq 0$, there is an $x \in E$ such that $0<$ $x \leqq|w|$; now if $U \in \mathscr{B}$ is such that $x \notin U, w \notin \widetilde{U}$.

(d) If $U \in \mathscr{B}, \varnothing \subset A \subseteq \widetilde{U}$, and $A \uparrow w$ in $C_{\infty}(X)$, then $w \in \widetilde{U}$. For suppose that $x \in E$ and that $|x| \leqq|w|$. Then

$$
\left\{y^{+}+w^{-}: y \in A\right\} \uparrow w^{+}+w^{-}=|w| \geqq|x|,
$$

so

$$
\left\{|x| \wedge\left(y^{+}+w^{-}\right): y \in A\right\} \uparrow|x|
$$

Now set

$$
B=\left\{z: z \in E, \exists y \in A, 0 \leqq z \leqq|x| \wedge\left(y^{+}+w^{-}\right)\right\} \text {• }
$$

Then $B \uparrow$, and as $E$ is orderdense in $C_{\infty}(X), B \uparrow|x|$. But if $z \in B$ there is a $y \in A$ such that

$$
z \leqq y^{+}+w^{-} \leqq y^{+}+y^{-}=|y|,
$$

so, as $y \in \widetilde{U}, z \in U$. Because $U \in \mathscr{B}, x \in U$. As $x$ is arbitrary, $w \in \widetilde{U}$.

(e) Consequently the sets $\widetilde{U} \cap H$ all satisfy the Fatou condition, and $\mathfrak{I}$ is Fatou. (Here we have used the fact that $H$ is orderdense in $C_{\infty}(X)$, so that if $A \uparrow w$ in $H$, then $A \uparrow w$ in $\left.C_{\infty}(X)\right)$.

(f) It also follows that $\mathfrak{I}$ is Levi. For suppose that $A \subseteq H$ is directed upwards, is not empty, and is bounded. Then of course $B=\left\{y^{+}: y \in A\right\}$ is directed upwards, and it is bounded because $\mathfrak{I}$ is locally solid. Now suppose that $x>0$ in $C_{\infty}(X)$. Let $U \in \mathscr{B}$ be such that $x \in \widetilde{U}$. Let $n>0$ be such that $A \leqq n U$. Now

$$
\left\{n^{-1} y \wedge x: y \in B\right\}
$$

is a subset of $\tilde{U}$, directed upwards; so its supremum belongs to $\widetilde{U}$ and cannot be $x$. Thus $\sup _{y \in B} y \wedge n x$ is not $n x$, and $B$ satisfies the condition of Proposition 1 ; so $B$, and therefore $A$, is bounded above in $C_{\infty}(X)$. Let $z_{0}=\sup A$ in $C_{\infty}(X)$; this exists as $C_{\infty}(X)$ is Dedekind complete. If $V \in \mathscr{B}$, there is an $m>0$ such that $m^{-1} A \subseteq \tilde{V}$, so by (d) again $m^{-1} z_{0} \in \tilde{V}$ i.e. $z_{0} \in m \tilde{V}$. As $V$ is arbitrary, $z_{0} \in H$, and is the required upper bound for $A$ in $H$. 
(g) Thus $\mathfrak{I}$ satisfies the conditions of Nakano's theorem, and $H$ is complete. So $\hat{E}$ may be regarded as the closure of $E$ in $H$. Because $E$ is orderdense in $H$, it is orderdense in $\hat{E}$. Finally, it is easy to see that the topology on $\hat{E}$ induced by $\mathfrak{T}$ is Fatou, because $\mathfrak{I}$ itself is Fatou and $\hat{E}$ is orderdense in $H$.

REMARK. Of course the condition "Archimedean" in the hypotheses of the theorem is redundant, because any Riesz space with a Hausdorff locally solid linear space topology must be Archimedean. The same applies to Theorem 2 below.

4. Counter-example. Suppose that $E=C([0,1])$, the space of real-valued continuous functions on the unit interval. Give $E$ the topology induced by \|\|$_{1}$ where

$$
\|x\|_{1}=\int|x| d \mu_{L} \forall x \in E,
$$

$\mu_{L}$ being Lebesgue measure. Then \|\|$_{1}$ is a Riesz norm so the topology is locally solid. But it is not Fatou and $E$ is not orderdense in its completion $L^{1}\left(\mu_{L}\right)$.

5. Lebesgue topologies. I should now like to proceed to a stronger condition, also fulfilled by many examples. Because it is of great interest in many contexts, I give as general a definition as I can. Let $E$ be any partially ordered set. A topology $\mathfrak{I}$ on $E$ is Lebesgue if, whenever $A$ is a non-empty subset of $E$ and either $A \uparrow x$ or $A \downarrow x$ in $E$, then $x$ belongs to the closure $\bar{A}$ of $A$. We shall be interested, of course, in linear space topologies on Riesz spaces; in this case, $\mathfrak{T}$ is Lebesgue iff $0 \in \bar{A}$ whenever $\varnothing \subset A \downarrow 0$.

Now the ordinary topologies on the $L^{p}$ spaces, for $0 \leqq p<\infty$, are Lebesgue; so is the norm topology on $c_{0}(N)$. We note that the exceptions are the $L^{\infty}$ and $C(X)$ spaces. However, the weak topology $\mathfrak{I}_{s}\left(L^{\infty}, L^{1}\right)$ is Lebesgue; in fact it is the case that the Mackey topology $\mathfrak{I}_{k}\left(L^{\infty}, L^{1}\right)$ is Lebesgue. Of course, if $\mathfrak{I}$ is Lebesgue and $\mathbb{B}$ is weaker than $\mathfrak{I}$, then $B$ is Lebesgue.

Lebesgue topologies have many remarkable properties. I give one of the simplest.

Lemma 1. A Lebesgue locally solid linear space topology on a Riesz space is Fatou.

Proof. Let $U$ be any neighbourhood of 0 ; let $V$ be a closed neighbourhood of 0 included in $U$; let $W$ be a solid neighbourhood of 0 included in $V$. The point is that $\bar{W}$ is solid ([4, Proposition IV. 
4.8]). But now $\bar{W} \subseteq U$ and $\bar{W}$ satisfies the Fatou condition because the topology is Lebesgue.

6. Theorem 2. Let $E$ be an Archimedean Riesz space with a Lebesgue locally solid Hausdorff linear space topology. Then the completion $\hat{E}$ of $E$ also has a Lebesgue topology.

Proof. We know by Lemma 1 and Theorem 1 that $E$ is orderdense in $\hat{E}$. Suppose, if possible, that $A \downarrow 0$ in $\hat{E}, A$ is not empty, but that $0 \notin \bar{A}$. Let $U$ be a solid neighbourhood of 0 in $\hat{E}$ such that $A$ does not meet $U$. Let $V$ be a solid neighbourhood of 0 in $\hat{E}$ such that $V+V+V \leqq U$. Fix $x_{0} \in A$ and find a $y_{0} \in E$ such that $x_{0}-y_{0} \in V$; without loss of generality, I may suppose that $y_{0} \geqq 0$. Now

$$
\left\{y_{0} \wedge\left(x_{0}-x\right)^{+} ; x \in A\right\} \uparrow y_{0} \wedge x_{0},
$$

so if

$$
B=\left\{z: z \in E, \exists x \in A, 0 \leqq z \leqq y_{0} \wedge\left(x_{0}-x\right)^{+}\right\} ，
$$

$B \uparrow x_{0} \wedge y_{0}$ in $\hat{E}$. Similarly,

$$
C=\left\{w: w \in E, 0 \leqq w \leqq\left(y_{0}-x_{0}\right)^{+}\right\} \uparrow\left(y_{0}-x_{0}\right)^{+},
$$

and so $B+C \uparrow y_{0}$ in $E$. As the topology on $E$ is Lebesgue, there exist $z \in B$ and $w \in C$ such that

$$
y_{0}-w-z \in V \text {. }
$$

But as $V$ is solid, $w \in V$, so $y_{0}-z \in V+V$, and

$$
x_{0}-z=y_{0}-z+\left(x_{0}-y_{0}\right) \in V+V+V \subseteq U \text {. }
$$

However, there is an $x \in A$ such that $0 \leqq z \leqq\left(x_{0}-x\right)^{+}$, and there is an $x_{1} \in A$ such that $x_{1} \leqq x \wedge x_{0} \leqq x_{0}-z$. But $U$ is solid, so $x_{1} \in U$; which is the contradiction we require.

7. Conclusion. I think that Theorem 1 is more surprising than Theorem 2. Both Fatou and Lebesgue topologies are frequently mysterious; but when we require a topology to be both locally solid and Lebesgue we are imposing such a powerful condition that we expect agreeable results to follow quickly. The Fatou property is harder to tackle. Its actual applications in Theorem 1, while certainly essential (see $\S 4$ ), are buried too deep in the argument to be readily disentangled; so it's not clear just what it is about Fatou topologies that makes the theorem true.

Theorem 1 is reminiscent of the result in [1] that if $E$ is any Riesz space, then the canonical image of $E$ in $E^{\times \times}$or $\left(E_{\tilde{n}}\right)_{\tilde{n}}$ is orderdense. 
In fact this can be deduced from Theorem 1, though (as far as I know) only by an extremely involved route. But there may be some hope that the techniques of [1] could be adapted to give a simpler proof of Theorem 1.

Theorem 2 is more straightforward, and can be proved independently of Theorem 1 without much difficulty. If in Theorem 2 we know that $E$ is locally convex, there is a proof direct from the result in [1] quoted above. But the hypothesis of local convexity doesn't seem to help in Theorem 1.

Theorem 2 recalls the construction of the ordinary function spaces. If the spaces $L^{1}, L^{2}$ etc. are thought of as completions of the space $S$ of equivalence classes of simple functions under the appropriate norms, their properties can be deduced from the fact that each of these norms induces a Lebesgue locally solid topology on $S$.

\section{REFERENCES}

1. W. A. J. Luxemburg and A. G. Zaanen, Notes on Banach function spaces $X$, Proc. Nat. Acad. Sci., Amsterdam (A) 67 (1964), 494-506; also in Indagationes Mathematicae, vol. 26.

2. R. Metzler and H. Nakano, Quasi-Norm Spaces, Trans. Amer. Math. Soc., 123 (1966), 1-31.

3. H. Nakano, Linear topologies on semi-ordered linear spaces, J. Fac. Sci. Hokkaido (I), 12 (1953), 87-104.

4. A. L. Peressini, Ordered Topological Spaces, Harper and Row, 1967.

5. H. H. Schaefer, Topological Vector Spaces, Macmillan, 1966.

6. B. Z. Vulikh, (trans. L. F. Boron) Introduction to the Theory of Partially Ordered Spaces, Wolters-Noordhoff, 1967.

Received April 1, 1971. This work was done while I held a Central Electricity Generating Board Junior Research Fellowship at Churchill College, Cambridge.

UNIVERSITY OF ESSEX

COLCHESTER, ENGLAND 



\section{PACIFIC JOURNAL OF MATHEMATICS}

\section{EDITORS}

\author{
H. SAMELSON \\ Stanford University \\ Stanford, California 94305 \\ C. R. HOBBY \\ University of Washington \\ Seattle, Washington 98105
}

\author{
J. DugundJI \\ Department of Mathematics \\ University of Southern California \\ Los Angeles, California 90007
}

\author{
RICHARD ARENS \\ University of California \\ Los Angeles, California 90024
}

\section{ASSOCIATE EDITORS}
E. F. BECKENBACH
B. H. NeumanN
F. WOLF
K. YoSHIDA

\section{SUPPORTING INSTITUTIONS}

\author{
UNIVERSITY OF BRITISH COLUMBIA \\ CALIFORNIA INSTITUTE OF TECHNOLOGY \\ UNIVERSITY OF CALIFORNIA \\ MONTANA STATE UNIVERSITY \\ UNIVERSITY OF NEVADA \\ NEW MEXICO STATE UNIVERSITY \\ OREGON STATE UNIVERSITY \\ UNIVERSITY OF OREGON \\ OSAKA UNIVERSITY
}

\author{
UNIVERSITY OF SOUTHERN CALIFORNIA \\ STANFORD UNIVERSITY \\ UNIVERSITY OF TOKYO \\ UNIVERSITY OF UTAH \\ WASHINGTON STATE UNIVERSITY \\ UNIVERSITY OF WASHINGTON \\ $* * * *$
$*$
AMERICAN MATHEMATICAL SOCIETY
NAVAL WEAPONS CENTER
}

The Supporting Institutions listed above contribute to the cost of publication of this Journal, but they are not owners or publishers and have no responsibility for its content or policies.

Mathematical papers intended for publication in the Pacific Journal of Mathematics should be in typed form or offset-reproduced, (not dittoed), double spaced with large margins. Underline Greek letters in red, German in green, and script in blue. The first paragraph or two must be capable of being used separately as a synopsis of the entire paper. The editorial "we" must not be used in the synopsis, and items of the bibliography should not be cited there unless absolutely necessary, in which case they must be identified by author and Journal, rather than by item number. Manuscripts, in duplicate if possible, may be sent to any one of the four editors. Please classify according to the scheme of Math. Rev. Index to Vol. 39. All other communications to the editors should be addressed to the managing editor, Richard Arens, University of California, Los Angeles, California, 90024.

50 reprints are provided free for each article; additional copies may be obtained at cost in multiples of 50 .

The Pacific Journal of Mathematics is published monthly. Effective with Volume 16 the price per volume (3 numbers) is $\$ 8.00$; single issues, $\$ 3.00$. Special price for current issues to individual faculty members of supporting institutions and to individual members of the American Mathematical Society: $\$ 4.00$ per volume; single issues $\$ 1.50$. Back numbers are available.

Subscriptions, orders for back numbers, and changes of address should be sent to Pacific Journal of Mathematics, 103 Highland Boulevard, Berkeley, California, 94708.

PUBLISHED BY PACIFIC JOURNAL OF MATHEMATICS, A NON-PROFIT CORPORATION

Printed at Kokusai Bunken Insatsusha (International Academic Printing Co., Ltd.), 270, 3-chome Totsuka-cho, Shinjuku-ku, Tokyo 160, Japan. 


\section{Pacific Journal of Mathematics}

\section{Vol. 43, No. 2 \\ April, 1972}

Arne P. Baartz and Gary Glenn Miller, Souslin's conjecture as a problem on the real line....................................... 277

Joseph Barback, On solutions in the regressive isols ............... 283

Barry H. Dayton, Homotopy and algebraic K-theory ................ 297

William Richard Derrick, Weighted convergence in length ............ 307

M. V. Deshpande and N. E. Joshi, Collectively compact and semi-compact sets of linear operators in topological vector spaces ............. 317

Samuel Ebenstein, Some $H^{p}$ spaces which are uncomplemented in $L^{p} \ldots . .327$

David Fremlin, On the completion of locally solid vector lattices ......... 341

Herbert Paul Halpern, Essential central spectrum and range for elements of

a von Neumann algebra............................... 349

G. D. Johnson, Superadditivity intervals and Boas' test ............. 381

Norman Lloyd Johnson, Derivation in infinite planes . . . . . . . . . . . 387

V. M. Klassen, The disappearing closed set property .............. 403

B. Kuttner and B. N. Sahney, On the absolute matrix summability of Fourier series ........................................... 407

George Maxwell, Algebras of normal matrices................... 421

Kelly Denis McKennon, Multipliers of type $(p, p) \ldots \ldots \ldots \ldots \ldots \ldots . . \ldots 29$

James Miller, Sequences of quasi-subordinate functions ............. 437

Leonhard Miller, The Hasse-Witt-matrix of special projective varieties ..... 443

Michael Cannon Mooney, A theorem on bounded analytic functions ...... 457

M. Ann Piech, Differential equations on abstract Wiener space .......... 465

Robert Piziak, Sesquilinear forms in infinite dimensions ............. 475

Muril Lynn Robertson, The equation $y^{\prime}(t)=F(t, y(g(t))) \ldots \ldots \ldots \ldots .483$

Leland Edward Rogers, Continua in which only semi-aposyndetic

subcontinua separate ............................... 493

Linda Preiss Rothschild, Bi-invariant pseudo-local operators on Lie

groups ...................................... 503

Raymond Earl Smithson and L. E. Ward, The fixed point property for

arcwise connected spaces: a correction ...................... 511

Linda Ruth Sons, Zeros of sums of series with Hadamard gaps .......... 515

Arne Stray, Interpolation sets for uniform algebras............... 525

Alessandro Figà-Talamanca and John Frederick Price, Applications of random Fourier series over compact groups to Fourier multipliers .. 